\title{
Function, Development and Challenges of COVID-19 Diagnostic Methods in Two Areas: RT-PCR Tests and Serology Tests
}

\author{
Ke $\mathrm{Diao}^{1, \dagger}$, and Tiancheng $\mathrm{Yu}^{2, \dagger}$ \\ ${ }^{1}$ School of Biological Sciences and Biotechnology, Beijing forestry University, No.35 East Qinghua Road, China \\ ${ }^{2}$ The Department of Medicine, Soochow university, No. 199, Ren'ai Road, China \\ †These two authors contributed equally.
}

\begin{abstract}
The ongoing outbreaks of Severe Acute Respiratory Syndrome Coronavirus 2 (SARS-CoV-2) has led to a worldwide pandemic of Coronavirus Disease (COVID-19) in 2019. Nucleic Acid Tests as the current most prevalent method are able to detect the presence of SARS-CoV-2 from infected patients by comparing target viral genome sequences in high sensitivity and accuracy. Three widely applied nucleic acid methods for clinical and research purpose including RT-PCR tests, LAMP and CRISPR-Cas based detection are introduced firstly, followed by the discussion of Antibody Tests, which are ICG and CL immunoassay tests.These two fields of COVID-19 diagnostic methods exhibit some advantages and drawbacks depending on various clinical settings. Antibody test is supplementary and complementary of other diagnostic methods while Nucleic acid tests are overall effectively and rapidly to diagnose infected patients for immediate treatment and isolation. The combination of these two methods may eventually control the dissemination of COVID-19 pandemic.
\end{abstract}

\section{Introduction}

\subsection{Nucleic Acid Tests and Serology/Antibody Tests}

In December 2019, an outbreak of pneumonia with unknown cause began in China's Hubei province, which caused global health problems due to its ease of transmission. For rapid diagnosis and control of highly infectious diseases, suspicious populations were isolated and diagnostic/treatment procedures were developed based on patient epidemiological and clinical data. After extensive studies published, a new severe acute respiratory syndrome coronavirus 2 (SARS-COV-2) was identified as the cause of the disease, labeled with COVID-19.

Nucleic acid testing is the primary method of diagnosing COVID-19, which offers the most sensitive and early detection of COVID-19. The nucleic acid test is to look for the presence of a novel corona virus in the respiratory tract specimen, blood or stool of a patient to determine whether the patient has been infected with a virus. In a very short time, scientists completed the analysis of the whole genome sequence of the novel Corona virus. They discovered the specific nucleic acid sequence in the novel corona virus by comparing it with the genome sequences of other species. Viral RNA needs to be first reversely transcribed into cDNA and then amplified for detection. PCR amplification and detection should use the fluorescence quantitative PCR instrument specified in the approved product specification. By the $\mathrm{Ct}$ value of the sample obtained by fluorescence quantitative PCR, it can be determined whether the patient sample contains a novel Corona virus [1]. Several commonly used nucleic acid detection methods are introduced, including Real-Time RT-PCR tests, LAMP and CRISPR-Cas based detection.

A serological test, or antibody test, is considered as an indispensable auxiliary technique to detect SARS-CoV-2 infection since detecting antibodies in blood samples is convenient, safe, economic and highly sensitive [2]. The antibody test is based on the antigen-antibodies interaction in patients' blood after symptoms of SARS-CoV-2 emerge, roughly around 14-21 days [3]. For asymptomatic and recovered patients, serological tests are ideal at point of care. Within 15 minutes, a simple point-of-care mass-produced lateral flow immunoassay testing kit can detect antibodies simultaneously against SARS-CoV-2 virus in human blood [4]. Current methods of serological test are responsible for sensing three types of Immunoglobulin in response to the immune system from two structural protein of SARS-CoV-2, which to be more specific are IgM (Immunoglobulin M), IgG (Immunoglobulin G), and IgA (Immunoglobulin A) released due to the foreign stimulus of viral particles spike (S) and nucleocapsid (N) proteins [5]. IgM and IgG Immunol responses to $\mathrm{S}$

*⿳亠口冖口. 
protein are regarded as the main research areas because of their unique physiological and clinical characteristics for vaccine treatment and diagnostic research to control the COVID-19 pandemic [6]. One of the serological tests, Enzyme-Linked Immunosorbent Assay (ELISA) is the standard and widely used method to measure the antibody level from blood serum and blood plasma, while other testing methods including, but not limited to, colloidal gold immunochromatographic assays, magnetic chemiluminescent immunoassays, and rapid test cassettes and dipsticks will also be discussed further in detail in terms of development, function and future expectation [7].

\subsection{Biological properties of SARS-CoV-2}

SARS-CoV-2 has a single strand forward RNA genome, which is about 30,000 nucleotides in length [8, 9]. These include RNA-dependent RNA polymerase (RdRP) and four structural proteins $[9,10]$. RdRP acts in conjunction with nonstructural proteins to maintain genome fidelity. A region of the RdRP gene in SARS-CoV-2 was shown to be highly similar to a region of the RdRP gene found in bat coronavirus RaTG13 and 96\% similar to the RaTG13 overall genome sequence [8].

The four structural proteins of SARS-CoV-2 include spike surface glycoprotein (S), small envelope protein $(\mathrm{E})$, matrix protein $(\mathrm{M})$ and nucleocapsid protein $(\mathrm{N})$. In coronaviruses, the $\mathrm{S}$ gene encodes receptors that bind to spike proteins in virus-infected cells. This spike protein mediates receptor binding and membrane fusion, which determines host tropism and transmissibility [11].

\subsection{Pathological features and clinical picture of SARS-CoV-2}

Patients with COVID-19 exhibit high viral loads in their upper and lower respiratory tract within 5 to 6 days after the onset of symptoms. A nasopharyngeal (NP) swab and/or an oropharyngeal (OP) swab are often recommended for screening or diagnosis of early infection [12-15]. Molecular diagnosis of the viral genome and serological blood tests are performed by RT-PCR, chest X-ray or CT scan. The most common laboratory abnormalities in RT-PCR in positive patients were lymphocytopenia, leukopenia, thrombocytopenia, elevated CRP and inflammatory markers, elevated cardiac biomarkers, decreased albumin, and abnormal renal and liver function [16].

\subsection{The severity of SARS-CoV-2}

The spread of SARS-CoV-2 was so effective that it led to the current COVID-19 (coronavirus Disease 2019) pandemic, which had killed more than 300,000 people worldwide by mid-May 2020. The highest morbi-mortality of COVID-19 is observed among older patients [17]. Patients in the intensive care unit (ICU) are older than patients not requiring ICU [18]. In a study using an observational database from 169 hospitals in Asia, Europe, and North America, an age of more than 65 years-old was associated with a higher risk of in-hospital death: $10.0 \%$ mortality rates for people $\geq 65$ years-old vs. $4.9 \%$ for those $<65$ years-old [19]. Frail older adults, especially those with comorbidities (such as high blood pressure, obesity, diabetes), have a higher risk of death if they become infected than younger adults.

\section{Nucleic Acid Testing}

Nucleic acid testing is considered as the gold standard method of diagnosing COVID-19. Nucleic-acid-based tests currently offer the most sensitive and early detection of COVID-19. Several commonly used nucleic acid detection methods are introduced in the following part, including RT-PCR, LAMP, and CRISPR-Cas based detection.

\subsection{Real-Time RT-PCR}

These tests utilize standard RT-PCR protocols, including cell lysis, nucleic acid extraction and purification, and multiple PCR amplification and detection with fluorescence signal readings. RT-PCR involves the reverse transcription of SARS-CoV-2 RNA into complementary DNA (cDNA) strands, which are then amplified to specific regions of the cDNA [20]. After the primers and probes were designed, PCR tests were performed. Resultant amplification of DNA subject to specific detection or analytical methods such as electrophoresis or sequencing.

Molecular diagnosis by nucleic acid amplification using RT-PCR has been the method of choice [8, 21]. The RdRp and $\mathrm{N}$ genes of the virus can be used for molecular detection of SARS-CoV-2 [22, 23]. Scientists did a test that gives confirmatory results within $45 \mathrm{~min}$ and can qualitatively detect the virus in different specimens, such as oropharyngeal/nasopharyngeal swabs, nasal wash, etc., or aspirates [10, 24]. More recently, RT-PCR method targets have included Orfla/b and the gene encoding the $\mathrm{S}$ protein.

The main advantage of RT-PCR analysis is that both amplification and analysis are performed in a closed system to minimize false-positive results associated with contamination of amplified products. However, an important issue with RT-PCR testing is the risk of triggering false negatives and false positives. Many of the "suspicious" cases have been reported to have typical clinical features of COVID-19 and the same specific computed tomography (CT) images [25].

Table 1 Comparison between PCR and loop-mediated isothermal amplification (LAMP) reactions [26, 27]

\begin{tabular}{|c|c|c|}
\hline $\begin{array}{l}\text { Amplification } \\
\text { method }\end{array}$ & $\begin{array}{l}\text { Sample } \\
\text { Preparation }\end{array}$ & $\begin{array}{l}\text { Experimental } \\
\text { steps }\end{array}$ \\
\hline
\end{tabular}




\begin{tabular}{|c|c|c|c|c|c|c|}
\hline PCR & $\begin{array}{l}\text { Thermal } \\
\text { cycling } \\
\text { (Multiple } \\
\text { heating and } \\
\text { cooling cycle; } \\
\text { hence, bulky } \\
\text { and } \\
\text { cumbersome). }\end{array}$ & $\begin{array}{l}\text { Always requires } \\
\text { sample } \\
\text { concentration and } \\
\text { preparation } \\
\text { (Time-consuming). }\end{array}$ & $\begin{array}{l}\text { Multiple } \\
\text { protocols } \\
\text { (Complicated } \\
\text { and requires } \\
\text { a skilled } \\
\text { technician). }\end{array}$ & $\begin{array}{l}\text { Inhibitors } \\
\text { hinder } \\
\text { the } \\
\text { reaction. }\end{array}$ & $\begin{array}{l}\text { Diagnostic } \\
\text { sensitivity } \\
(95 \%) \text { is } \\
\text { currently } \\
\text { reported } \\
\text { lower than } \\
\text { LAMP }\end{array}$ & $\begin{array}{l}\text { Established } \\
\text { technique. }\end{array}$ \\
\hline LAMP & $\begin{array}{l}\text { Isothermal } \\
\text { and } \\
\text { continuous } \\
\text { amplification } \\
\text { (Smaller, } \\
\text { simpler, } \\
\text { hence } \\
\text { portable). }\end{array}$ & $\begin{array}{l}\text { For virus } \\
\text { detection, for } \\
\text { example, influenza } \\
\text { or human } \\
\text { norovirus, LAMP } \\
\text { assay offers } \\
\text { one-step detection. } \\
\text { Sample } \\
\text { preparation steps } \\
\text { are simplified. }\end{array}$ & $\begin{array}{l}\text { Single } \\
\text { protocol } \\
\text { (Faster). }\end{array}$ & $\begin{array}{l}\text { Tolerate } \\
\text { inhibitors } \\
\text { and more } \\
\text { stable. }\end{array}$ & $\begin{array}{l}\text { Diagnostic } \\
\text { sensitivity }> \\
95 \% .\end{array}$ & $\begin{array}{l}\text { Applications } \\
\text { using } \\
\text { LAMP } \\
\text { assays are } \\
\text { being } \\
\text { explored. }\end{array}$ \\
\hline
\end{tabular}

\subsection{Loop-mediated isothermal amplification}

Many organizations are eager to use isothermal nucleic acid amplification technology for SARS-CoV-2 POCT, thus avoiding the need for expensive thermal cyclers. Loop-mediated isothermal amplification (LAMP) may be the most promising alternative to PCR because of its many advantages in specificity, sensitivity, reaction efficiency and product yield [28]. Loop mediated isothermal amplification (LAMP) is a novel isothermal nucleic acid amplification method. LAMP exhibits increased sensitivity and specificity due to an exponential amplification feature that utilizes 6 different target sequences simultaneously identified by separate distinct primers in the same reaction [29]. LAMP assays are significantly rapid, and do not require expensive reagents or instruments, which aids in cost reduction for coronavirus detection [30].

As shown in Table 1, in contrast to PCR, the reaction is carried out at a constant temperature. LAMP simplifies DNA with high specificity, efficiency, and rapidity under isothermal conditions. This method uses a set of four specially designed primers, and DNA polymerase with strand displacement activity to synthesize target DNA up to 109 copies in less than an hour at a constant temperature of $65{ }^{\circ} \mathrm{C}$ [31]. Studies have shown that the technique has good sensitivity and specificity [32]. studied 76 nasopharyngeal swab samples from suspected COVID-19 patients; the sensitivity and specificity were $100 \%$ and $97.6 \%$ respectively. In another study, sensitivity and specificity of the technique were both $100 \%$ compared to RT-PCR [33]. Franklin Wang-Ngai Chow reported developing and evaluating a rapid and simple one-step colorimetric reverse transcriptional loop-mediated isothermal amplification (RT-LAMP) assay COVID-19-LAMP, for detection of SARS-CoV-2. RT-LAMP combines reverse transcriptase, DNA polymerase, $\mathrm{pH}$ indicator, and six primers to amplify RNA templates [34-36]. COVID-19-LAMP Assay can detect SARS-CoV-2 with a low detection limit and ss highly sensitive and specific for detection of SARS-CoV-2 in clinical samples [37].

\subsection{CRISPR-Cas based detection}

Recently, researchers have proposed a rapid detection method for coronavirus based on the CRISPR/Cas system. CRISPR/Cas are archaea and bacterial adaptive immune systems against foreign genetic components such as phages.

In this technique, the programmable protein attaches to the target via a guide RNA used to cut the target sequence. Several types of Cas proteins have different properties. Among them, Cas9 gets more attention in gene editing, while Cas12a and Cas13a are more effective in diagnosing diseases [26, 27]. Cas 12a is DNA specific, but Cas13a can be used with RNA to facilitate the detection of SARS-CoV-2. Zhang et al. reported that the specific highly sensitive enzyme reported Unlocking (SHERLOCK) technology is a CRISPR/Cas13 nucleic acid-based rapid detection of SARS-CoV -2.

They targeted the $\mathrm{S}$ and ORF1ab proteins in the coronavirus genome. Cas13 identifies and binds to previously identified target sequences, resulting in a fairly random cleavage of surrounding ssRNA molecules. SHERLOCK technology USES quenched fluorescent ssRNA to report molecules. The presence of the ssRNA coronavirus genome in the sample activates Cas13, resulting in a quantifiable signal. Amplification of target DNA or RNA by recombinase polymerase amplification (RPA) or reverse transcriptase -RPA (RTRPA) prior to the initiation of the reaction improves the sensitivity of the assay. The amplified DNA is then converted to RNA through a combination of RPA and T7 transcription. Finally, simultaneous incorporation of ssRNA reporter gene (Biotin-RNA-FITC) was used for detection.CRISPR/Cas13-based diagnosis is much faster and more sensitive than detection by RT-qPCR [38].

\subsection{Future expectations}

Compared these methods with CT scan, although CT scan sensitivity is high (98\%) with low specificity, it may aid but not replace molecular methods for the diagnosis and is considered an important tool to diagnose complications and to assess the severity of the disease in 
order to improve the quality of care [39, 40]. And CT scans are not effective in diagnosing patients who are asymptomatic or presymptomatic, or who have mild symptoms without pneumonia [41]. The limitation of CT scanning is only available in large hospitals; in addition, two radiologists need to check images.

Significant progress has been made in the detection and determination of SARS-CoV-2, which has played an important role in controlling the COVID-19 pandemic. However, the low detection sensitivity of SARS-CoV-2 RNA POCT, especially using unextracted RNA samples, reduces the screening accuracy in symptomatic and asymptomatic patients with low viral load. In addition, the longer time required to detect SARS-CoV-2 does not facilitate rapid and on-site screening of suspected COVID-19 patients. Therefore, there is an urgent need to develop a fast and simple method for the highly efficient release and enrichment of SARS-CoV-2 RNA.

\section{Serology/Antibody test}

\subsection{Comparison to RT-PCR}

Comparing to RT-PCR test, antibody diagnosis, or serological diagnosis, can also act as an indispensable auxiliary tool to detect SARS-CoV-2 infection. Detection of the SARS-CoV-2-specific antibody in the blood sample is convenient, safe and highly sensitive [42]. Also, a large number of asymptomatic patients who have infected SARS-CoV-2 may not be found and PCR-tested, making antibody tests crucial when diagnosing COVID-19 [43]. What is more, serological tests have high efficacy. Within merely 15 minutes, a simple point-of-care mass-produced lateral flow immunoassay testing kit that can detect immunoglobulin $\mathrm{M}(\mathrm{IgM})$ and $\operatorname{IgG}$ antibodies simultaneously against SARS-CoV-2 virus in human blood within 15 minutes. It is appropriate to modify patient's status in different infection stages [44]. Affordable and transportable, serological application using, such as lateral flow essay and chemiluminescence, are especially crucial for diagnosis in remote area or patients who present mild to moderate illness, and some might be cheaper and easier to implement at the point of care [45].

\subsection{Principle of Antibody test}

Belonging to the same Orthocoronavirinae subfamily, in immunological aspects, SARS-CoV-19 shares relative same traits as its relatives: MERS-CoV and SARS-CoV. Spreading through the respiratory tract contact (droplets, respiratory secretions, saliva, and direct contact), the patients' immunological react can be detected more accurately after two weeks [18]. While aiming for higher antigenicity, antibody tests mainly focusing on spike surface glycoprotein $(\mathrm{S})$ and nucleocapsid proteins $(\mathrm{N})$ [46-48]. Current research focusing on three types of Immunoglobulin related to these two proteins, which are IgM (Immunoglobulin M), IgG (Immunoglobulin G) and IgA (Immunoglobulin A), for the former two have developed proven technique, helping researchers making useable testing methods in less time [42]. While the latter IgA has been discovered an ELISA testing method with high accuracy [49]. According to an SRAS infection pattern, the first week after infection, IgM levels expect to increase, then peak at two weeks, and gradually reduce to near-background levels. Like IgA, IgM is detectable after 8 days, gradually reaching its peak level in three weeks then maintained constantly at a high level for a long time, even more than 48 days[49, 50]. When applying these theories to commercially produced ELISA testing kits, IgG and IgM shows higher specificity than IgA, but lower sensitivity [51].

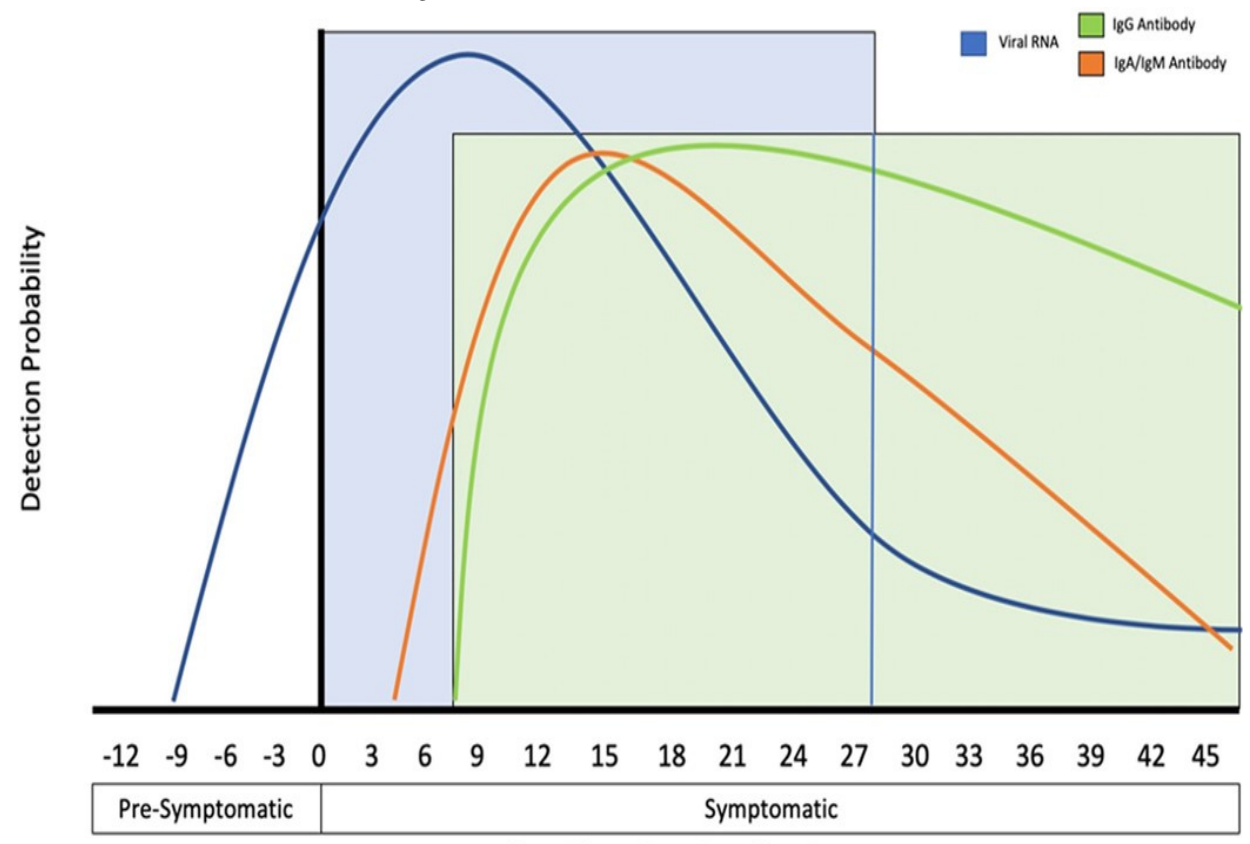

Days Since Symptom Onset

Figure 1. Viral RNA and antibodies (IgA, IgM, and IgG) detection probability in days since symptom of SARS-cov-2 onset. The testing windows of nucleic acid amplification tests (RT-PCR, blue) and serology tests (antibody, green) are indicated [52]. 


\subsection{Clinical and Research Applications}

Focusing on practical applications, antibodies test kits have entered the global market. Their testing methods include, but are not limited to, colloidal gold immunochromatographic assays, magnetic chemiluminescent immunoassays, enzyme-linked immunosorbent assays (ELISA), and rapid test cassettes and dipsticks [53]. An ideal serological test for SARS-CoV-2 should have a high diagnostic sensitivity, low or no cross-reactivity with other existing antibodies, and a high sample throughput to prevent the delay of timely therapeutic decisions due to false-negative results [54]. Research teams worldwide have developed varieties of antigen detection kits, which are awaiting approval from the Administration in each country for massive production. Montesinos's research group evaluated two chemiluminescence and three lateral flow immunoassays for the diagnosis of COVID-19. Two chemiluminescence immunoassays: Roche Elecsys ${ }^{\circledR}$ Anti-SARS-CoV-2 Test and Abbott SARS-CoV-2 IgG, and three lateral flow immunoassays: Wondfo
SARS-CoV-2 Antibody Test, ASK COVID-19 IgG/IgM Rapid Test, and Dynamiker 2019-nCoV IgG/IgM Rapid Test. All test kits showed high diagnostic sensitivities and over $90 \%$ diagnostic specificity [55]. Here we focus on two mainstream POC serological applications: colloidal gold-based immunochromatographic strip assay and chemiluminescence immunoassay.

\subsubsection{Colloidal gold-based immunochromatographic (ICG) strip assay}

Colloidal gold particles are nanometer-sized aqueous gold suspension, in large accumulation, they will appear purplish-red color. Commonly, when chloroauric acid ( $\mathrm{HAuCl} 4)$ is reduced with sodium citrate dilute solutions, colloidal gold particles will be driven to form small aggregates that will associate with antibodies. These gold particles of specific sizes are commonly used for multiple label experiments to be isolated and differentiated microscopically [56]. Colloidal gold-labeled antibodies are widely used in electron microscopy, and can be used for light microscopy with additional steps [57].
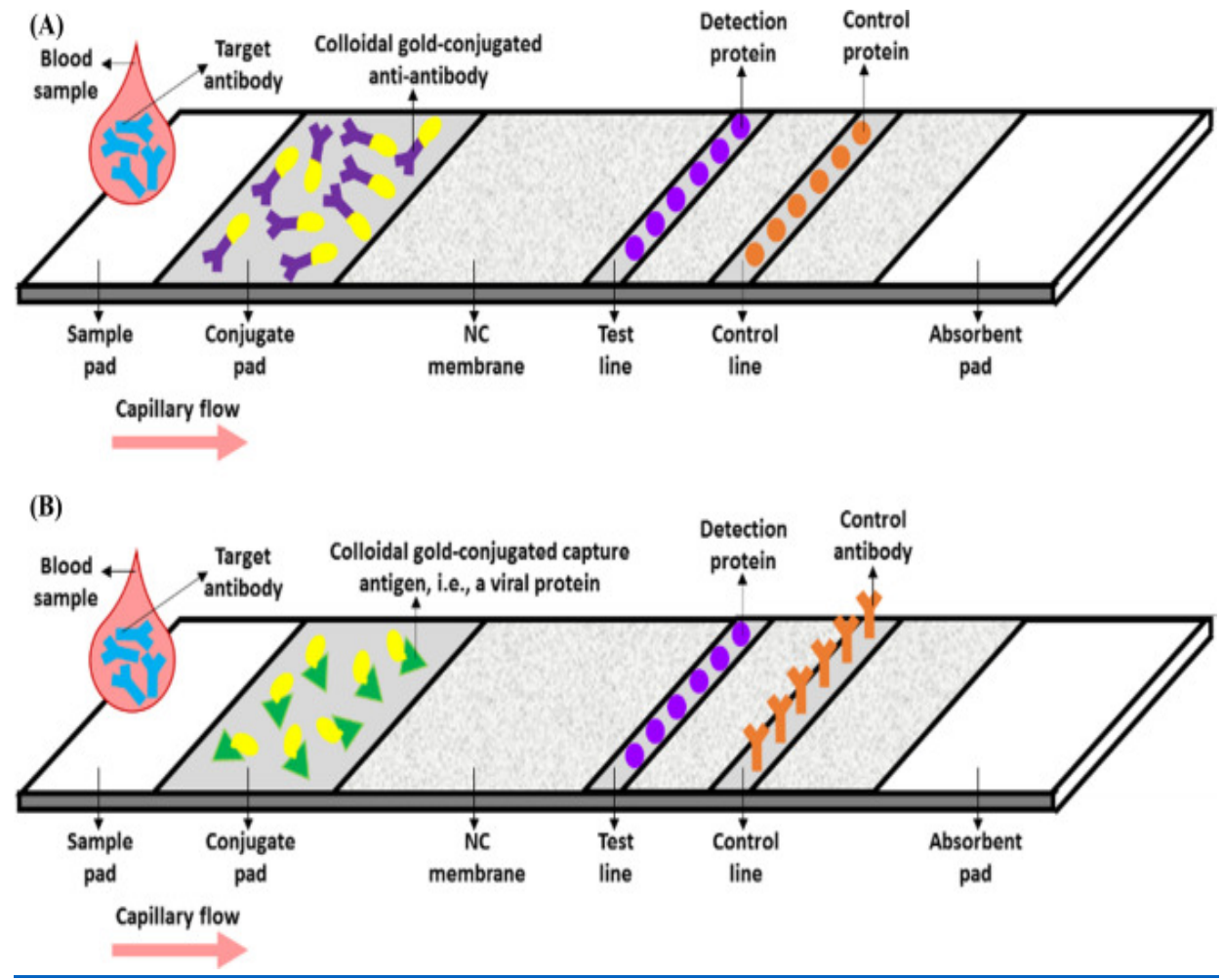

Figure 2. (A) and (B) Schematic diagram and interpretation standard of the SARS-COV-2 antibody detection kit. [58].

A recently published study reported the latest clinical practice results at RenMin Hospital of Wuhan University in the first-line diagnosis and treatment of 2019-nCoV. In that study, 19 patients with negative nucleic acid tests but with clinical symptoms and CT imaging features of COVID-19 were serologically tested. The results reported that 16 patients $(84.21 \%)$ were positive for 2019-nCoV IgM antibodies and 18 patients (94.74\%) were positive for 2019-nCoV IgG antibodies. The majority of the 2019-nCoV IgM and IgG antibody detection assays have high clinical specificity and clinical detection rates, and they should be used to confirm the status of nucleic acid detection negative samples. Furthermore, in terms of treatment monitoring and disease progression, the decline and disappearance of 2019-nCoV IgM concentration and the rise of 2019-nCoV IgG concentration indicated that patients gradually recovered and produced immunity to pathogenic 2019-nCoV [59]. 
Colloidal gold-antibody conjugates are easy to prepare and are an excellent choice for microscopic applications. These gold-nanoparticles are commonly used in a variety of immunoassay testing methods for years, for their outstanding stability, simple manufacturable sizes and brilliant compatibility with antibodies and antigens, as well as other biological molecules. Colloidal gold immunochromatography test of $\mathrm{IgM} / \mathrm{IgG}$ offers the advantages of widely used, (whole blood, serum and plasma can be detected), simple operation (no professional technical personnel required for equipment), fast detection (10-15 minutes for the whole process) and low cost [61]. Meanwhile testing kits using IgA has been developed. It is predicted that future testing kit might combine three antibodies to make better diagnosis [49].

\subsubsection{Chemiluminescence (CL) immunoassay}

Though the colloidal gold method makes diagnosis very convenient, it still has drawn backs, highlighting CL immunoassay. The protein-antibody conjugations are difficult to manipulate thus they may have inconsistent binding affinities. When the antibodies are small, it is difficult to discover and accumulate, which block the tool to be built from the first stage [61].

Without an external light sources or other operation, Chemiluminescence (CL) is the emission of luminescence that results from a chemical reaction. CL assay has significant advantages such as high sensitivity, low detection limits, wide linear dynamic ranges, simple instrumentation, low cost and good speed of response [62].

Chemiluminescence detection of $\operatorname{IgM}$ antibody showed a positive conversion earlier (about 1-2 days earlier), positive conversion rates higher in different stages of the disease, and a trend of declining positive rate later than colloidal gold method. For IgG antibodies, the chemiluminescence method showed a positive conversion earlier and the positive rate climbing more quickly than the colloidal gold method. No obvious negative-converting tendency of $\mathrm{IgG}$ detection was observed within 35 days after the onset of disease. Although colloidal gold method is generally less sensitive than chemiluminescence method, it shows the advantages of shorter turn-around time, more simple procedure, and no special equipment required. The two methodologies can be chosen according to different laboratory conditions [63].

As a quantitative testing method, serological diagnosis shows a high-efficiency low time-cost. Colloidal gold is suitable for point-of-care diagnoses while chemiluminescence is proper for research [64]. It is certain that both mainstream testing methods, colloidal gold and Chemiluminescence, have their drawbacks. For colloidal gold may requires massive production of antibodies to combine with gold particles [65]. While chemiluminescence require trained professionals to operate the steps $[65,66]$. It is our belief that with the mass production of antibodies, both methods will be more precise and less expensive.

\section{Conclusion}

In conclusion, asymptomatic infected persons are currently the focus of attention of the infected population. If nucleic acid speed of nucleic acid can be improved and the cost can be reduced to enable rapid screening of asymptomatic infected persons, unnecessary losses can be greatly reduced. In the future, whether we can devise a way to detect all asymptomatic infected people will be crucial to getting rid of the virus completely.

Antibody detection has incomparable advantages over nucleic acid detection in the sampling process and detection time. The nucleic acid test is used to detect if the subject has the virus, while the antibody test is used for supplementary testing or combined nucleic acid test in suspected patients with negative nucleic acid test results. These two kinds of detection methods have their characteristics and it is best to use them together.

We conclude that many molecular and serological technologies have been developed to date, but that there is still an urgent need for safe, rapid, affordable, more sensitive and accurate methods to diagnose SARS-induced infection - COV-2 that can control the spread of COVID-19.

diagnoses. It is inevitable for serological tests to have the possibility to have false-negative results. One reason might be the low concentrations of antibodies. It is clear that when antibodies are lower than the detection levels, results may be negative. Either relate to the specific time collecting samples-antibodies may not produce at first week or decrease after a long time. Another reason may be highly related to patients' different immune response antibody production. For future expectations, test kits using $\operatorname{IgA}$, IgM and IgG as diagnosis methods might be put into commercial production for higher perception. The combination of these three antibodies may reduce the chances of false-negative results. The failure or success of treatment cannot be determined, as the antigen may persist following appropriate antiviral treatment. It is also very difficult to achieve rapid detection of whole virus antigens as antigen detection usually requires high sensitivity and antigen contents in samples are generally low. Research on recognizing antigens and tracking antibodies requires time, as does the development of monoclonal antibodies, which are the required raw materials for immunological detection against viruses.

\section{Reference}

1. M. N. Esbin, et al., Overcoming the bottleneck to widespread testing: a rapid review of nucleic acid testing approaches for COVID-19 detection. Rna, ((2020)). 26(7): p. 771-783.

2. N. Zhu, et al., A Novel Coronavirus from Patients with Pneumonia in China, 2019. N Engl J Med, ((2020)). 382(8): p. 727-733.

3. D. S. Ojeda, et al., Emergency response for evaluating SARS-CoV-2 immune status, seroprevalence and convalescent plasma in 
Argentina. PLoS Pathog, (2021). 17(1): p. e1009161.

4. M. Michel, et al., Evaluating ELISA, Immunofluorescence, and Lateral Flow Assay for SARS-CoV-2 Serologic Assays. Front Microbiol, (2020). 11: p. 597529.

5. M. Chen, et al., Clinical applications of detecting IgG, IgM, or IgA antibody for the diagnosis of COVID-19: A meta-analysis and systematic review. Int J Infect Dis, (2021).

6. Y. Galipeau, et al., Humoral Responses and Serological Assays in SARS-CoV-2 Infections. Front Immunol, (2020). 11: p. 610688.

7. Y. Uwamino, et al., Evaluation of the usability of various rapid antibody tests in the diagnostic application for COVID-19. Ann Clin Biochem, (2021): p. 4563220984827.

8. P. Zhou, et al., A pneumonia outbreak associated with a new coronavirus of probable bat origin. Nature, (2020). 579(7798): p. 270-273.

9. A. Wu, et al., Genome Composition and Divergence of the Novel Coronavirus (2019-nCoV) Originating in China. Cell Host Microbe, (2020). 27(3): p. 325-328.

10. N. R. Sexton, et al., Homology-Based Identification of a Mutation in the Coronavirus RNA-Dependent RNA Polymerase That Confers Resistance to Multiple Mutagens. J Virol, (2016). 90(16): p. 7415-7428.

11. D. Wrapp, et al., Cryo-EM structure of the 2019-nCoV spike in the prefusion conformation. Science, (2020). 367(6483): p. 1260-1263.

12. L. Zou, et al., SARS-CoV-2 Viral Load in Upper Respiratory Specimens of Infected Patients. $N$ Engl J Med, (2020). 382(12): p. 1177-1179.

13. C. Kim, et al., Comparison of nasopharyngeal and oropharyngeal swabs for the diagnosis of eight respiratory viruses by real-time reverse transcription-PCR assays. PLoS One, (2011). 6(6): p. e21610.

14. P. K. Chan, et al., Laboratory diagnosis of SARS. Emerg Infect Dis, (2004). 10(5): p. 825-31.

15. I. Paranjpe, et al., Clinical Characteristics of Hospitalized Covid-19 Patients in New York City. medRxiv, (2020).

16. J. Zhu, et al., Clinicopathological characteristics of 8697 patients with COVID-19 in China: a meta-analysis. Fam Med Community Health, (2020). $8(2)$.

17. Z. Wu and J. M. McGoogan, Characteristics of and Important Lessons From the Coronavirus Disease 2019 (COVID-19) Outbreak in China: Summary of a Report of 72314 Cases From the Chinese Center for Disease Control and Prevention. Jama, (2020). 323(13): p. 1239-1242.

18. D. Wang, et al., Clinical Characteristics of 138 Hospitalized Patients With 2019 Novel
Coronavirus-Infected Pneumonia in Wuhan, China. JAMA, (2020). 323(11): p. 1061-1069.

19. M. R. Mehra, et al., Cardiovascular Disease, Drug Therapy, and Mortality in Covid-19. N Engl J Med, (2020). 382(25): p. e102.

20. W. M. Freeman, S. J. Walker, and K. E. Vrana, Quantitative RT-PCR: pitfalls and potential. Biotechniques, (1999). 26(1): p. 112-22, 124-5.

21. F. Wu, et al., A new coronavirus associated with human respiratory disease in China. Nature, (2020). 579(7798): p. 265-269.

22. C. Huang, et al., Clinical features of patients infected with 2019 novel coronavirus in Wuhan, China. Lancet, (2020). 395(10223): p. 497-506.

23. W. Zhang, et al., Molecular and serological investigation of 2019-nCoV infected patients: implication of multiple shedding routes. Emerg Microbes Infect, (2020). 9(1): p. 386-389.

24. F. Wolters, et al., Multi-center evaluation of cepheid xpert(R) xpress SARS-CoV-2 point-of-care test during the SARS-CoV-2 pandemic. J Clin Virol, (2020). 128: p. 104426.

25. Y. Wang, et al., Combination of RT-qPCR testing and clinical features for diagnosis of COVID-19 facilitates management of SARS-CoV-2 outbreak. $J$ Med Virol, (2020). 92(6): p. 538-539.

26. S. Y. Li, et al., CRISPR-Cas12a-assisted nucleic acid detection. Cell Discov, (2018). 4: p. 20.

27. C. Myhrvold, et al., Field-deployable viral diagnostics using CRISPR-Cas13. Science, (2018). 360(6387): p. 444-448.

28. T. Ji, et al., Detection of COVID-19: A review of the current literature and future perspectives. Biosens Bioelectron, (2020). 166: p. 112455.

29. T. Notomi, et al., Loop-mediated isothermal amplification of DNA. Nucleic Acids Res, (2000). 28(12): p. E63.

30. M. Shen, et al., Recent advances and perspectives of nucleic acid detection for coronavirus. J Pharm Anal, (2020). 10(2): p. 97-101.

31. K. Nagamine, T. Hase, and T. Notomi, Accelerated reaction by loop-mediated isothermal amplification using loop primers. Mol Cell Probes, (2002). 16(3): p. 223-9.

32. Y. Kitagawa, et al., Evaluation of rapid diagnosis of novel coronavirus disease (COVID-19) using loop-mediated isothermal amplification. J Clin Virol, (2020). 129: p. 104446.

33. C. Yan, et al., Rapid and visual detection of 2019 novel coronavirus (SARS-CoV-2) by a reverse transcription loop-mediated isothermal amplification assay. Clin Microbiol Infect, (2020). 26(6): p. 773-779.

34. S. H. Lee, et al., One-Pot Reverse Transcriptional Loop-Mediated Isothermal Amplification (RT-LAMP) for Detecting MERS-CoV. Front Microbiol, (2016). 7: p. 2166. 
35. S. F. Hu, et al., Development of reverse-transcription loop-mediated isothermal amplification assay for rapid detection and differentiation of dengue virus serotypes 1-4. BMC Microbiol, (2015). 15: p. 265.

36. P. Rajko-Nenow, et al., A rapid RT-LAMP assay for the detection of all four lineages of Peste des Petits Ruminants Virus. J Virol Methods, (2019). 274: p. 113730 .

37. F. W. Chow, et al., A Rapid, Simple, Inexpensive, and Mobile Colorimetric Assay COVID-19-LAMP for Mass On-Site Screening of COVID-19. Int J Mol Sci, (2020). 21(15).

38. M. Dara and M. Talebzadeh, CRISPR/Cas as a Potential Diagnosis Technique for COVID-19. Avicenna J Med Biotechnol, (2020). 12(3): p. 201-202.

39. P. Dashraath, et al., Coronavirus disease 2019 (COVID-19) pandemic and pregnancy. Am J Obstet Gynecol, (2020). 222(6): p. 521-531.

40. P. G. Wasilewski, et al., COVID-19 severity scoring systems in radiological imaging - a review. Pol $J$ Radiol, (2020). 85: p. e361-e368.

41. F. Cui and H. S. Zhou, Diagnostic methods and potential portable biosensors for coronavirus disease 2019. Biosens Bioelectron, (2020). 165: p. 112349.

42. Z. Huang, et al., Characteristics and roles of severe acute respiratory syndrome coronavirus 2-specific antibodies in patients with different severities of coronavirus 19. Clin Exp Immunol, (2020). 202(2): p. 210-219.

43. D. O. Andrey, et al., Diagnostic accuracy of Augurix COVID-19 IgG serology rapid test. Eur $J$ Clin Invest, (2020). 50(10): p. e13357.

44. B. Shen, et al., Clinical evaluation of a rapid colloidal gold immunochromatography assay for SARS-Cov-2 IgM/IgG. Am J Transl Res, (2020). 12(4): p. 1348-1354.

45. N. Sethuraman, S. S. Jeremiah, and A. Ryo, Interpreting Diagnostic Tests for SARS-CoV-2. JAMA, (2020). 323(22): p. 2249-2251.

46. A. C. Walls, et al., Structure, Function, and Antigenicity of the SARS-CoV-2 Spike Glycoprotein. Cell, (2020). 183(6): p. 1735.

47. B. Sun, et al., Kinetics of SARS-CoV-2 specific IgM and IgG responses in COVID-19 patients. Emerg Microbes Infect, (2020). 9(1): p. 940-948.

48. X. Chi, et al., A neutralizing human antibody binds to the N-terminal domain of the Spike protein of SARS-CoV-2. Science, (2020). 369(6504): p. 650-655.

49. M. Infantino, et al., Closing the serological gap in the diagnostic testing for COVID-19: The value of anti-SARS-CoV-2 IgA antibodies. $J$ Med Virol, (2020).

50. A. Padoan, et al., IgA-Ab response to spike glycoprotein of SARS-CoV-2 in patients with
COVID-19: A longitudinal study. Clin Chim Acta, (2020). 507: p. 164-166.

51. N. M. A. Okba, et al., Severe Acute Respiratory Syndrome Coronavirus 2-Specific Antibody Responses in Coronavirus Disease Patients. Emerg Infect Dis, (2020). 26(7): p. 1478-1488.

52. C. H. Chau, J. D. Strope, and W. D. Figg, COVID-19 Clinical Diagnostics and Testing Technology. Pharmacotherapy, (2020). 40(8): p. 857-868.

53. S. J. R. da Silva, et al., Clinical and Laboratory Diagnosis of SARS-CoV-2, the Virus Causing COVID-19. ACS Infect Dis, (2020). 6(9): p. 2319-2336.

54. C. W. Farnsworth and N. W. Anderson, SARS-CoV-2 Serology: Much Hype, Little Data. Clin Chem, (2020). 66(7): p. 875-877.

55. S. Y. Chen, et al., Multicenter evaluation of two chemiluminescence and three lateral flow immunoassays for the diagnosis of COVID-19 and assessment of antibody dynamic responses to SARS-CoV-2 in Taiwan. Emerg Microbes Infect, (2020). 9(1): p. 2157-2168.

56. E. A. Berg and J. B. Fishman, Labeling Antibodies Using Colloidal Gold. Cold Spring Harb Protoc, (2020). 2020(4): p. 099333.

57. Y. Pan, et al., Serological immunochromatographic approach in diagnosis with SARS-CoV-2 infected COVID-19 patients. J Infect, (2020). 81(1): p. e28-e32.

58. S. Dowlatshahi, E. Shabani, and M. J. Abdekhodaie, Serological assays and host antibody detection in coronavirus-related disease diagnosis. Arch Virol, (2021).

59. N. Li, et al., Molecular diagnosis of COVID-19: Current situation and trend in China (Review). Exp Ther Med, (2020). 20(5): p. 13.

60. G. Yong, et al., Evaluation of the auxiliary diagnostic value of antibody assays for the detection of novel coronavirus (SARS-CoV-2). J Med Virol, (2020). 92(10): p. 1975-1979.

61. Y. Nasiri Khonsari and S. Sun, Recent trends in electrochemiluminescence aptasensors and their applications. Chem Commun (Camb), (2017). 53(65): p. $9042-9054$.

62. Y. Sun and J. Lu, Chemiluminescence-based aptasensors for various target analytes. Luminescence, (2018). 33(8): p. 1298-1305.

63. J. Y. Choe, et al., Diagnostic performance of immunochromatography assay for rapid detection of $\mathrm{IgM}$ and $\mathrm{IgG}$ in coronavirus disease 2019. J Med Virol, (2020). 92(11): p. 2567-2572.

64. S. Lijia, et al., Serological chemiluminescence immunoassay for the diagnosis of SARS-CoV-2 infection. J Clin Lab Anal, (2020). 34(10): p. e23466. 
65. S. Kaneko, et al., Clinical validation of an immunochromatographic SARS-Cov-2 IgM/IgG antibody assay with Japanese cohort. J Med Virol, (2020).

66. I. Selingerova, et al., Interpretive discrepancies caused by target values inter-batch variations in chemiluminescence immunoassay for SARS-CoV-2 IgM/IgG by MAGLUMI. J Med Virol, (2020). 